\title{
Successful Treatment with Colestimide for a Bout of Cholestasis in a Japanese Patient with Benign Recurrent Intrahepatic Cholestasis Caused by ATP8B1 Mutation
}

\author{
Satoko Uegaki ${ }^{1}$, Atsushi Tanaka ${ }^{1}$, Yosuke Mori ${ }^{2}$, Hiroko Kodama ${ }^{2}$, \\ Toshio Fukusato ${ }^{3}$ and Hajime Takikawa ${ }^{1}$
}

\begin{abstract}
Benign recurrent intrahepatic cholestasis (BRIC) is an autosomal recessive disorder characterized by bouts of cholestasis that resolve spontaneously without leaving considerable liver damage. Most of BRIC patients have mutations in $A T P 8 B 1$ gene coding FIC1 protein. It has been suggested that an imbalance between the gut absorption of bile acids and the liver excretion possibly causes the development of cholestasis. We encountered a Japanese woman patient with familial intrahepatic cholestasis type 1 (FIC1) deficiency manifesting BRIC, in whom a rapid and gross elevation of serum total bile acid (TBA) level preceded that of serum total bilirubin level. Interestingly, the early administration of colestimide prevented the development of hyperbilirubinemia along with the additional elevation of serum TBA level. This case suggests that FIC1 deficiency causes an imbalance between the gut absorption of bile acids and the liver excretion leading to cholestasis, and raised the possibility that colestimide may be used as an optional treatment for BRIC.
\end{abstract}

Key words: BRIC, cholestasis, FIC1, colestimide

(Inter Med 47: 599-602, 2008)

(DOI: 10.2169/internalmedicine.47.0534)

\section{Introduction}

Benign recurrent intrahepatic cholestasis (BRIC) is an autosomal recessive disorder characterized by episodic cholestasis without elevation of serum gamma-glutamyltransferase (GGT) levels, and usually lacks progressive liver damage (1). Recent studies have demonstrated that BRIC, or BRIC type 1, and another hereditary cholestatic disorder, progressive familial intrahepatic cholestasis type 1 (PFIC1), are caused by mutations in $A T P 8 B 1$ encoding FIC1 protein, P-type ATPase (2). We encountered a woman case with BRIC, in whom elevation of serum total bile acids (TBA) preceded clinical jaundice. She was successfully treated with colestimide, a negative ion exchange resin that prevents the absorption of bile acids from the intestine. In this report, we describe her clinical course with and without this treatment.
The possible contributions of farnesoid $\mathrm{X}$ receptor (FXR), a nuclear receptor regulating bile acid transport system, to the development of cholestasis or hyperbilirubinemia $(3,4)$ is also discussed.

\section{Case Report}

A Japanese woman, now aged 20 years old, was normally born to healthy parents with no consanguinity. She was entirely healthy until she developed jaundice for the first time at the age of 7 months old that lasted for 2 months. Although the jaundice resolved spontaneously, she developed the bouts of cholestasis at the ages of 5, 15 and 17 years that lasted for 1-2 months. Liver biopsy was performed at 9 months and 4 years old, with and without jaundice, respectively, and no chronic liver damage including fibrosis was found. She had no family history of any liver diseases. At

${ }^{1}$ Department of Medicine, Teikyo University School of Medicine, Tokyo, ${ }^{2}$ Department of Pediatrics, Teikyo University School of Medicine, Tokyo and ${ }^{3}$ Department of Pathology, Teikyo University School of Medicine, Tokyo

Received for publication August 17, 2007; Accepted for publication January 5, 2008

Correspondence to Dr. Atsushi Tanaka, a-tanaka@med.teikyo-u.ac.jp 


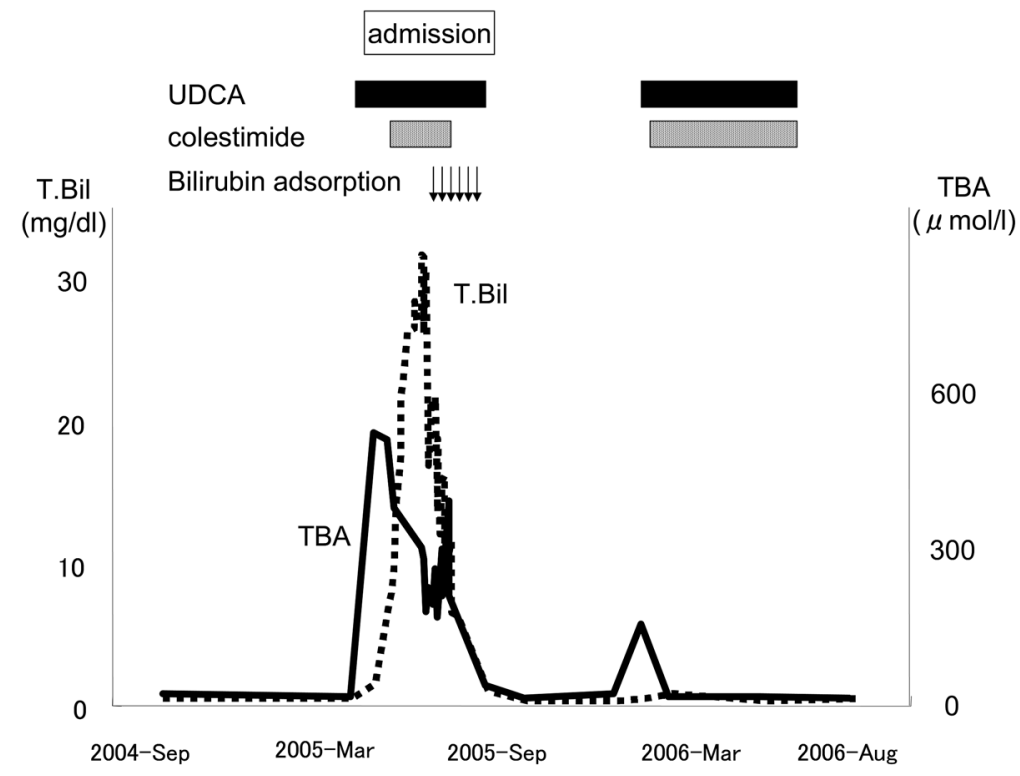

Figure 1. The recent clinical course of the present patient. Solid line, TBA. Dotted line, total bilirubin. Please note that the peak of TBA (solid line) proceeded the peak of total bilirubin, which was elevated even after the serum level of TBA began to fall. In contrast, the development of jaundice was prevented after the second peak of TBA, by administration of colestimide.

Table 1. The Peak Value of Serum TBA and Total Bilirubin in Four Episodes of Jaundice in the Clinical Course of the Patient

\begin{tabular}{|l|l|l|l|l|}
\hline & \multicolumn{2}{|c|}{ TBA } & \multicolumn{2}{c|}{ total bilirubin } \\
\hline & peak value & date & peak value & date \\
& $(\mu \mathrm{mol} / \mathrm{l})$ & & $(\mathrm{mg} / \mathrm{dl})$ & \\
\hline At 5 years old & 495 & $1991 / 10 / 31$ & 5.9 & $1991 / 11 / 15$ \\
\hline At 15 years old & 894 & $2001 / 10 / 10$ & 35.7 & $2002 / 1 / 9$ \\
\hline At 17 years old & 409 & $2003 / 6 / 19$ & 19.7 & $2003 / 6 / 30$ \\
\hline At 19 years old & 557 & $2005 / 4 / 22$ & 31.8 & $2005 / 6 / 7$ \\
\hline
\end{tabular}

age 19, she developed marked jaundice again and was admitted to our hospital.

On admission, total and direct bilirubin was 8.0 and 5.7 $\mathrm{mg} / \mathrm{dl}$, respectively. Serum GGT level was normal, 19 IU/l, and serum TBA was highly elevated at $542 \mu \mathrm{mol} / 1$. The recent clinical profile of this patient was shown in Fig. 1. Serum TBA level, although elevated up to $557 \mu \mathrm{mol} / \mathrm{l}$ at two weeks before admission, rapidly decreased after admission with administration of ursodeoxycholic acid (UDCA) and colestimide, negative ion exchange resin which has the adsorptive effect of bile acids in the intestine. By contrast, serum total bilirubin level was increased even after serum level of TBA began to fall, irrespective of treatment with UDCA and colestimide, and reached at the peak of $31.8 \mathrm{mg} /$ $\mathrm{dl}$ one month after admission. Therefore, we started a series of bilirubin adsorption, which were in turn very effective for the reduction of jaundice. Interestingly, by closer observation of her clinical course since her early episodes of jaundice, we noticed that the accumulation of TBA in sera always proceeded the development of marked jaundice by two weeks to 3 months (Table 1). Indeed, it has been previously demonstrated that an increased serum TBA level is followed by an increase of bilirubin in patients with BRIC (5).

To confirm the diagnosis of BRIC, we analyzed the $A T P 8 B 1$ gene of this patient. Genomic DNA was extracted from whole peripheral blood of the patient, and the ATP8B1 gene was amplified by polymerase chain reaction (PCR) using oligonucleotides, designed according to the sequence of the ATP8B1 from GenBank (AC027097). Amplified PCR products were subcloned and sequenced using Applied Bio- 


\begin{tabular}{|c|c|c|}
\hline & $\begin{array}{l}\text { Mutation detected } \\
\text { in the patient }\end{array}$ & Wild-type \\
\hline Exon 9 & $\begin{array}{ccc}305 & 306 & 307 \\
\text { TTC } & \text { CGC } & \text { CAC } \\
\text { Phe } & \text { Arg } & \text { His }\end{array}$ & $\begin{array}{ccc}305 & 306 & 307 \\
\text { TTC } & \text { TGC } & \text { CAC } \\
\text { Phe } & \text { Cys } & \text { His }\end{array}$ \\
\hline Exon 21 & $\begin{array}{ccc}866 & 867 & 868 \\
\text { TGC } & \text { C } & \text { GTC } \\
\text { Cys } & \text { His } & \text { Val }\end{array}$ & $\begin{array}{ccc}866 & 867 & 868 \\
\text { TGC } & \text { CGC } & \text { GTC } \\
\text { Cys } & \text { Arg } & \text { Val }\end{array}$ \\
\hline
\end{tabular}

Figure 2. The results of sequencing analysis. The patient had compound heterozygous mutations in exons 9 and 21.

systems 3,730xl genetic analysis system (Applied Biosystems, Foster City, CA, USA). Sequencing analyses of all exons revealed compound heterozygous and novel mutations in exons 9 and 21 (Fig. 2), amplified using oligonucleotides; 5'; TGA AGA ACC CAA TAA CCG ACT, and 3'; TGA ACG TAC CTG CAA AAA TGA for exon 9, and 5'; AGC AGC GGC AGA AAA ACT T, and 3'; CTC TTC ACC AGG TCC ACC AC for exon 21. In exon 9, we detected a missense mutation, TGC to CGC, resulting in amino acid substitution, Cys306Arg. Also in exon 21, a missense mutation, CGC to CAC, was found, leading to Arg867His. Neither of the mutations has been reported previously.

Recently it has been assumed that mutations of FIC1 gene in BRIC may inhibit nuclear translocation of FXR, which results in dysregulation of membrane transporters and the increased bile acids pool and jaundice $(3,4)$. To confirm this hypothesis, we performed immunostaining of FXR in the liver of the patient to locate the FXR protein in hepatocytes. The biopsied liver specimens had been obtained three times, at age 0 and 15 with deep jaundice and at age 4 without jaundice. Immunostaining of FXR was performed using an indirect immunoperoxidase method (EnVision kit, Dako Cytomation, Glostrup, Denmark). Anti-human FXR monoclonal antibody (clone; A9033A; PERSEUS PROTEOMICS Co., Ltd., Japan) and anti-human FXR polyclonal antibody (Santa Cruz, CA, USA) were used at a dilution of 1:100 and 1:200, respectively. The results of immunohistochemical studies shown in Fig. 3 demonstrate the significant accumulation of FXR protein in the nucleus in all specimens, irrespective of the presence of jaundice. As controls, we performed FXR immunostaining using liver specimens from patients without any liver disease and with obstructive jaundice. The results were quite similar to those with BRIC; strong nuclear accumulations of FXR were noted in both specimens (data not shown).

\section{Discussion}

BRIC is characterized by episodic jaundice without leaving any chronic liver damage. Recently BRIC type 2 (BRIC 2) caused by another mutational change in $A B C B 11$ gene has been demonstrated, and BRIC caused by ATP8B1 observed in the current patient is termed BRIC type 1 (BRIC1)

(6). The ATP8B1 gene encodes $140-\mathrm{kD}$ FIC1 protein, the P-type ATPase spanning the cell membrane (1). However, it

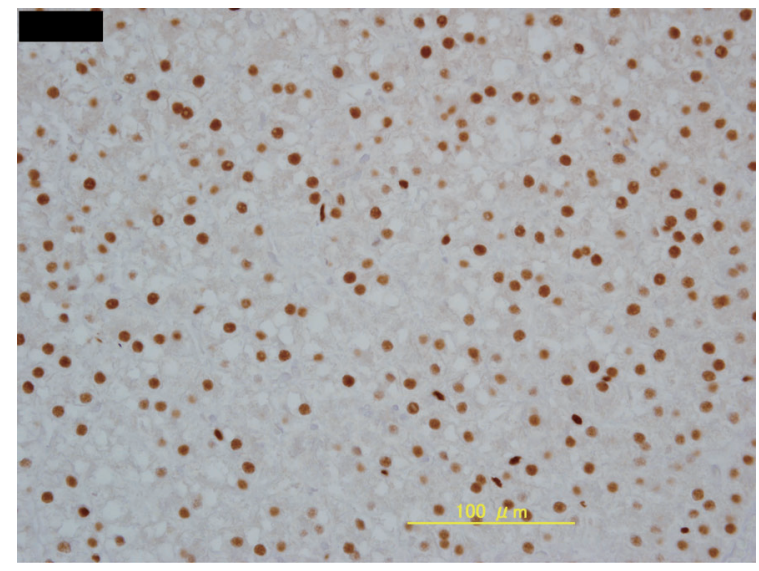

Figure 3. Immunostaining of FXR using the liver specimen obtained at age 15 with marked jaundice. FXR was deeply accumulated in hepatocellular nuclei. Similar strong nuclear staining was also shown in the liver specimens at age 0 and at age 15, without and with jaundice, respectively (not shown).

is still unclear how this genetic defect of ATP8B1 gene results in the intrahepatic cholestatic phenotype in BRIC. In this regard, Chen et al proposed that FIC1 protein mediates nuclear targeting of FXR through PKC-dependent phosphorylation of FXR $(3,4)$, which, in turn, prevents the accumulation of bile acid pool through downregulation of ASBT (apical sodium-dependent bile acid transporter) in the intestine and upregulation of BSEP (bile salt export pump) in hepatocytes. Therefore, it could be assumed that deterioration of FIC1 function caused by mutations in BRIC inhibit nuclear translocation of FXR, which results in upregulation of ASBT as well as downregulation of BSEP, eventually leading to the increased bile acids pool and jaundice.

To examine this possibility we performed immunostaining of FXR in the liver of the patient with BRIC. As shown, however, FXR was strongly accumulated in the nuclei of hepatocytes, even when jaundice was present (Fig. 3), and apparently this continuous accumulation in the nuclei is not coincident with the hypothesis. Nevertheless, nuclear targeting of FXR may not always be hampered in patients with BRIC. Rather, deterioration of FIC1 may transiently inhibit nuclear translocation of FXR, which would be enough for deregulation of membrane transporters and expansion of bile acid pool. Indeed, a recent report demonstrated that FXR expression in the liver of a patient with BRIC was downregulated at the protein level (7), suggesting that FXR might be mistargeted and largely degraded in BRIC. In addition, it is noteworthy that, when the serum bile acid level was augmented, development of jaundice was prevented by absorption of bile acids with colestimide administered before development of jaundice (Fig. 1). This therapeutic effect of colestimide supports the important role of the accumulation of bile acids prior to development of jaundice in the pathogenesis of BRIC. In fact, the cholestatic effect of bile acids, taurocholate and taurochenodeoxycholate, in rats has been frequently demonstrated $(8,9)$, and probably would arise from downregulation of hepatic membrane transporter, mul- 
tidrug resistance protein (Mrp2), at least in cholestasis induced by administration of taurolithocholic acid (10).

In conclusion, we encountered a case with BRIC, in whom several episodes of periodical jaundice developed. The precedence of increased bile acid pool prior to jaundice, along with successful prevention of jaundice with absorption of intestinal bile acids, could be interpreted as evidence sup- porting the hypothetical underlying mechanism of BRIC, deregulation of FXR and transporters in the intestine and liver resulting from mutated $A T P 8 B 1$. Colestimide, which prevented absorption of intestinal bile acids and was effective in this patient, may be used as an optional treatment for BRIC.

\section{References}

1. Luketic V, Shiffman M. Benign recurrent intrahepatic cholestasis. Clin Liver Dis 8: 133-149, 2004.

2. Bull L, van Eijk M, Pawlikowska L, et al. A gene encoding a Ptype ATPase mutated in two forms of hereditary cholestasis. Nat Genet 18: 219-224, 1998.

3. Chen F, Ananthanarayanan M, Emre S, et al. Progressive familial intrahepatic cholestasis, type 1 , is associated with decreased farnesoid X receptor activity. Gastroenterology 126: 756-764, 2004.

4. Frankenberg T, Chen F, Li X, et al. FIC1 (ATP8B1) mediates protein kinase dependent phosphorylation and nuclear targeting of the farnesoid X-receptor. Hepatology 44: 240A, 2006.

5. Bijleveld C, Vonk R, Kuipers F, Havinga R, Fernandes J. Benign recurrent intrahepatic cholestasis: a long-term follow-up study of two patients. Hepatology 9: 532-537, 1989.

6. van Mil S, van der Woerd W, van der Brugge G, et al. Benign recurrent intrahepatic cholestasis type 2 is caused by mutations in ABCB11. Gastroenterology 127: 379-384, 2004.
7. Nagasaka H, Chiba H, Hui S, et al. Depletion of high-density lipoprotein and appearance of triglyceride-rich low-density lipoprotein in a Japanese patient with FIC1 deficiency manifesting benign recurrent intrahepatic cholestasis. J Pediatr Gastroenterol Nutr 45: 96-105, 2007.

8. Kitani K, Kanai S. Tauroursodeoxycholate prevents taurocholate induced cholestasis. Life Sci 30: 515-523, 1982.

9. Tsukahara K, Kanai S, Ohta M, Kitani K. Taurine conjugate of ursodeoxycholate plays a major role in the hepatoprotective effect against cholestasis induced by taurochenodeoxycholate in rats. Liver 13: 262-269, 1993.

10. Beuers U, Bilzer M, Chittattu A, et al. Tauroursodeoxycholic acid inserts the apical conjugate export pump, Mrp2, into canalicular membranes and stimulates organic anion secretion by protein kinase C-dependent mechanisms in cholestatic rat liver. Hepatology 33: 1206-1216, 2001.

(C) 2008 The Japanese Society of Internal Medicine http://www.naika.or.jp/imindex.html 